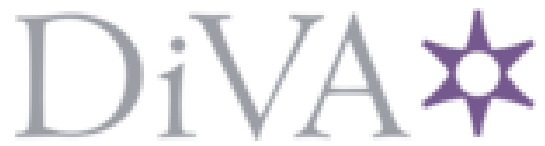

http://www.diva-portal.org

Postprint

This is the accepted version of a paper presented at 2018 48th European Microwave Conference (EuMC).

Citation for the original published paper:

Smirnov, S., Anoshkin, I V., Lioubtchenko, D., Oberhammer, J. (2018)

Carbon Nanotube Layer Modeling for Computer Simulation of Optically Controlled Phase Shifters

In: (pp. 827-830). IEEE

https://doi.org/10.23919/EuMC.2018.8541431

N.B. When citing this work, cite the original published paper.

Permanent link to this version:

http://urn.kb.se/resolve?urn=urn:nbn:se:kth:diva-239891 


\title{
Carbon Nanotube Layer Modeling for Computer Simulation of Optically Controlled Phase Shifters
}

\author{
Serguei Smirnov, Ilya V. Anoshkin, Dmitri V. Lioubtchenko, Joachim Oberhammer \\ Department of Micro and Nanosystems, KTH Royal Institute of Technology, Stockholm, Sweden \\ sergueis@kth.se
}

\begin{abstract}
We propose an approach for efficient modeling of thin carbon nanotube layers for full-wave device simulations without increasing the number of simulation mesh cells. A surface impedance, used in computer simulations, is calculated from the dielectric constant of the material. The dielectric constant is modeled by a Drude-Lorentz resonance, fitted to experimental results. The approach allowed to study the nanotube-induced losses and finite-size resonance effects in optically-controlled, dielectric rod waveguide-based phase shifters. The correctness of the model was verified both by the simulated and the measured S-parameters in the W-band.
\end{abstract}

Keywords - carbon nanotubes, millimeter waves, numerical simulation, optically controlled phase shifter, surface impedance.

\section{INTRODUCTION}

Modeling and design of waveguide components at millimeter-wave and Terahertz $(\mathrm{THz})$ frequencies meet an increasing interest for a number of applications. Those are high-speed telecommunications, radar systems, biomedical imaging and diagnosis, and material spectroscopy [1]-[4].

Hollow rectangular metal waveguides are the most used in the millimeter-wave frequency region, but their applications are limited due to metal conduction losses. Instead, the dielectric rod waveguide (DRW) is a promising platform for integrated $\mathrm{THz}$ and sub-THz systems. Made of high-permittivity dielectric material, DRWs offer only dielectric losses, which are considerably lower than those for the conventional metal waveguides [5]. Several components based on the DRWs have been proposed up to date such as passive waveguide couplers [6], antennas and antenna arrays [7], and traveling-wave amplifiers [8].

Recently, single-walled carbon nanotubes (SWCNTs) started to be extensively studied and have shown great potential for $\mathrm{THz}$ applications [9]. SWCNTs, together with other nanomaterials such as nanowires and nanoparticles, are usually simulated individually or at the small scale from the quantum mechanics approach [10], [11]. However, for their integration in electronic and optoelectronic devices, the typical electromagnetic field solver approach is needed. In that case, individual nanotube modeling is not possible with current state of the art simulation software and hardware, where the processor and memory usage are the limiting factors. Therefore, these nanomaterials are better represented as a homogeneous medium with effective electrical and mechanical properties that can be extracted from experimental data.
In this paper, we propose the modeling of thin SWCNT layers by a complex, frequency-dependent surface impedance. This approach permits not only to model the losses due to the finite conductance of the nanotube layer, but also its impact on the wave propagation when integrated with dielectric waveguides. This also allows studying any phase-shifting effects in the DRW generated by surface impedance variations. Moreover, this method does not require fine meshing to model thin SWCNT layers, and thus reduces the simulation complexity by keeping the number of mesh cells low. This results in fast simulations times and allows to perform optimization algorithms for the design of electrically-big devices.

\section{NumERicAl Simulations}

\section{A. Dielectric Constant}

The frequency-dependent, complex dielectric constant of a homogenous SWCNT layer is modeled by a Drude-Lorentz oscillator [12]:

$$
\varepsilon(\omega)=\varepsilon_{\infty}-\frac{\omega_{p D}^{2}}{\omega^{2}+j \Gamma_{D} \omega}+\frac{\omega_{p L}^{2}}{\omega_{o L}^{2}-\omega^{2}-j \Gamma_{L} \omega},
$$

where $\varepsilon_{\infty}$ is the high-frequency dielectric constant, $j$ is the imaginary unit and $\omega$ is the angular frequency. The parameters $\omega_{p D}$ and $\Gamma_{D}$ are respectively the plasma frequency and the damping constant of the Drude oscillator. The parameters $\omega_{p L}$, $\omega_{o L}$ and $\Gamma_{L}$ are the oscillator strength, the center frequency and the damping constant of the Lorentz oscillator.

The first frequency-dependent term on the right-hand side in (1) represents the free-electron model in metallic SWCNTs, following a Drude response. The exponential relaxation below the plasma frequency $\omega_{p D}$ leads to high losses at low frequencies. The second frequency-dependent term on the right-hand side in (1) represents a Lorentzian resonance. This term describes the plasmon resonance effect with the central frequency dependent on the finite length of the nanotubes [13], [14]. The Drude-Lorentz oscillator in this work was fitted to experimental measurements, with the initial values taken from [15] and [16]. The resulting parameters are summarized in Table 1, while a graphical representation of the complex dielectric constant in the $67-110 \mathrm{GHz}$ frequency range is shown in Fig. 1. 
Table 1. Drude-Lorentz Oscillator Parameters in $\mathrm{cm}^{-1}$

\begin{tabular}{lccc}
\hline Oscillator & $\omega_{p}$ & $\Gamma$ & $\omega_{0}$ \\
\hline Drude & 2292 & 67.2 & - \\
Lorentz & 101.3 & 0.114 & 2.33 \\
\hline
\end{tabular}

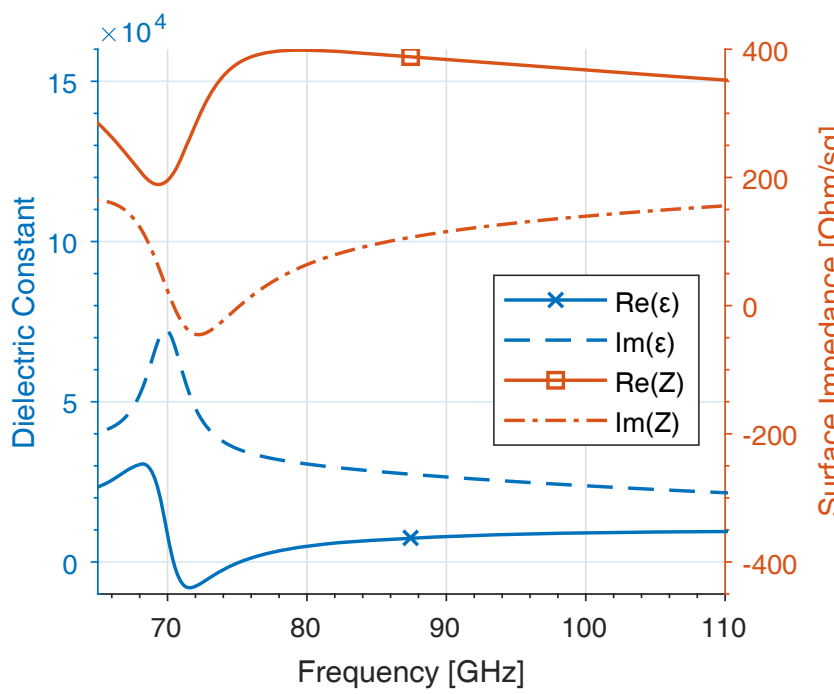

Fig. 1. Drude-Lorentz oscillator model of the thin SWCNT layer - Real and imaginary parts of the dielectric constant and of the surface impedance.

\section{B. Surface Impedance}

The surface impedance $Z(\omega)$ is defined as the relation between the tangential electric and magnetic fields, $\vec{E}_{t}$ and $\vec{H}_{t}$, at the interface of two media:

$$
\vec{E}_{t}(\omega)=Z(\omega) \cdot \vec{H}_{t}(\omega) \times \vec{n},
$$

where $\vec{n}$ is the normal vector to the interface. In the case of a dielectric material, the surface impedance can be written as function of the complex dielectric constant as following:

$$
Z(\omega)=\frac{-1}{j \omega t \cdot\left(\varepsilon(\omega)-\varepsilon_{h} \varepsilon_{0}\right)},
$$

where $t$ is the thickness of the SWCNT layer, $\varepsilon_{0}$ is the vacuum permittivity and $\varepsilon_{h}$ is the dielectric constant of the surrounding medium (vacuum) [15]. The calculated surface impedance is also shown in Fig. 1.

\section{Simulation Model}

Full-wave simulations were performed with CST Microwave Studio 2017. A sapphire DRW with a total length of $30 \mathrm{~mm}$, including $6 \mathrm{~mm}$-long symmetrical tapers in the $E$-plane on both ends and a $1 \times 0.5 \mathrm{~mm}^{2}$ cross-section, was excited by two standard metallic WR-10 waveguide ports as shown in Fig. 2.

The SWCNT layer was placed on top of the DRW, modeled as a tabulated surface impedance material with the complex impedance values calculated from (3). The layer's thickness was taken to be $t=30 \mathrm{~nm}$, corresponding to the measured sample with an optical transparency of $80 \%$. Laser excitation of the nanotubes was modeled as an impedance

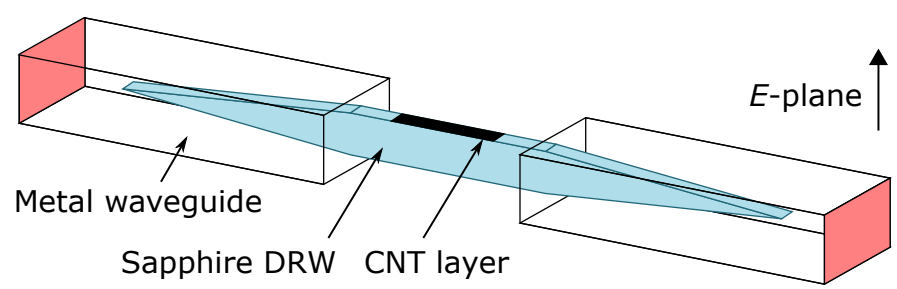

Fig. 2. Schematic drawing of the simulated and measured setup. A sapphire rectangular DRW is supported between two metal WR-10 waveguide ends for two-port S-parameter measurements. The rod's top surface is covered with a thin SWCNT layer, modeled by a surface impedance.
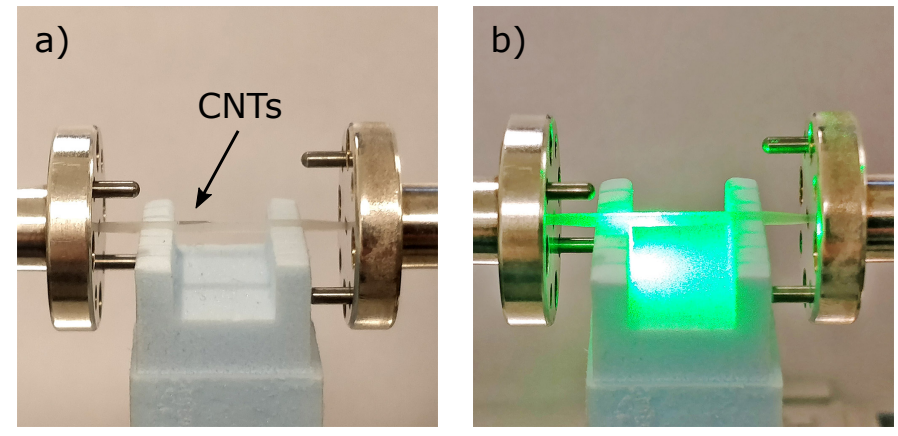

Fig. 3. Image of the measurement setup: a) in the initial state and b) the SWCNT layer is directly illuminated by a green laser.

variation, explained by an optically induced carbon nanotube polarization, corresponding to a capacitance decrease under illumination as was reported in [17].

\section{MEASUREMENT RESULTS AND DISCUSSION}

\section{A. Measurement Setup}

The sapphire rod was mechanically supported and inserted in WR-10 metal waveguides, according to the simulation model, as shown in Fig. 3. The SWCNT layer, with approximate dimensions of $5 \times 0.5 \mathrm{~mm}^{2}$ and with an optical transparency of $80 \%$, was manually applied from a nitrocellulose filter onto the top surface of the DRW by dry transfer, as illustrated in Fig. 4. Full two-port S-parameter measurements were performed with a Rohde \& Schwarz ZVA-24 Vector Network Analyzer with W-band extension head modules.

The measured $S_{21}$ and $S_{11}$ parameters are shown in Fig. 5 for an empty DRW and the same rod loaded with the SWCNT layer. The presence of nanotubes introduces high insertion loss in the lower frequency range as expected due to the Drude conductivity component, with a peak around $72 \mathrm{GHz}$ from the Lorentz oscillator. Good agreement is seen between the simulated and the measured values, with globally higher insertion loss for the measured ones. This is attributed to imperfections in the sapphire rod, such as micro-cracks and edge dents not considered in the simulations, as well as misalignment of the manually transferred SWCNT layer.

The measured return loss was also larger than the simulated, even for an empty DRW. This is mostly explained by defects in the rod and not perfectly symmetrical tapers due to sapphire machining difficulty. The misalignment of the 


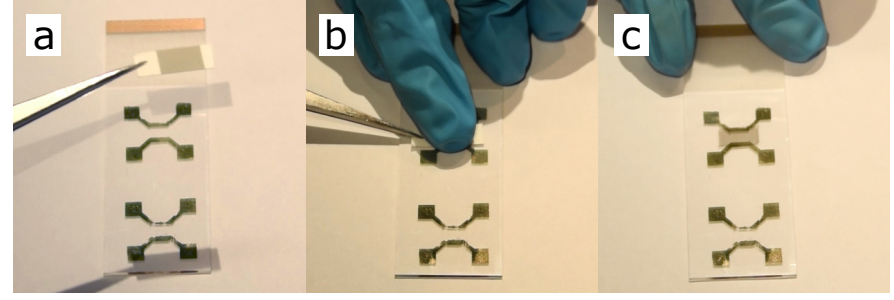

Fig. 4. Images of the SWCNT layer dry transfer method: a) the SWCNTs on a nitrocellulose filter, b) manual transfer by applying pressure, and c) the nanotube layer remains on the device.
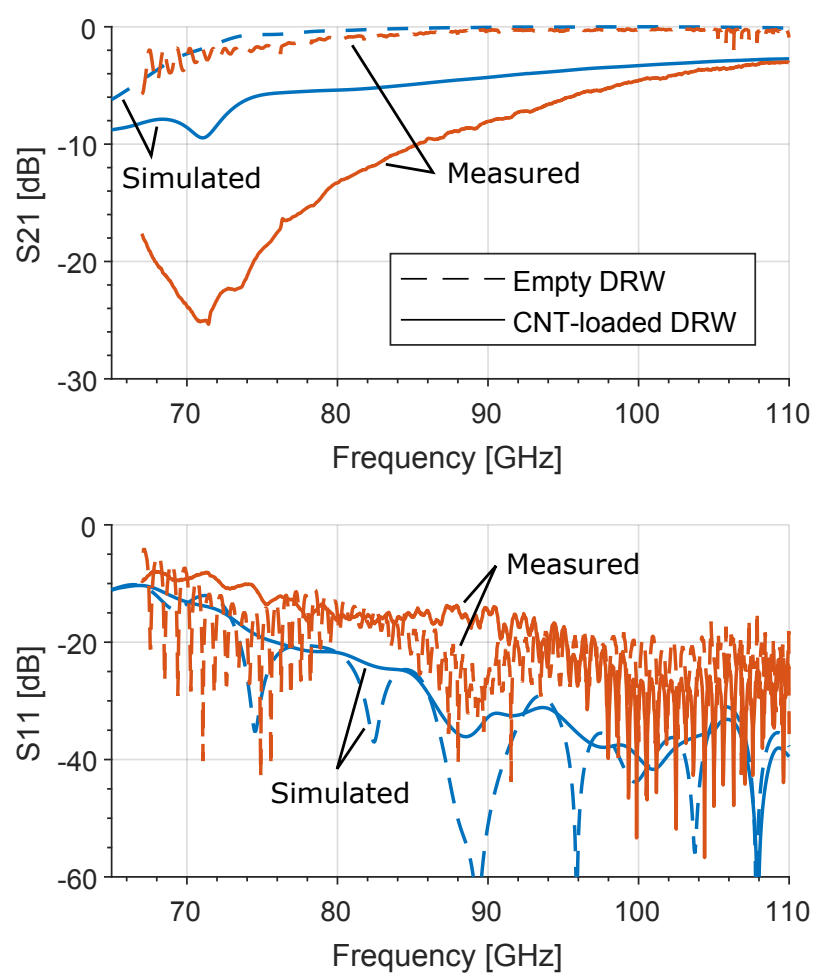

Fig. 5. Measured (red curves) and simulated (blue curves) S-parameters of an empty DRW (dashed curves) and of the same rod loaded with the SWCNT layer (plain curves).

DRW with respect to the metal waveguides due to manual positioning increases the return loss as well, and impacts the repeatability of the measurements.

\section{B. Phase Shifter}

The nanotube layer was then illuminated by a green laser source with a wavelength of $532 \mathrm{~nm}$. The light intensity of the laser was determined $\approx 9 \mathrm{~mW} / \mathrm{mm}^{2}$, measured with a "Sper Scientific 840011" laser power meter. The S parameters were measured with and without the illumination. The difference in their amplitude was negligible $(<0.5 \mathrm{~dB})$, and the laser-illuminated values are therefore not presented in Fig. 5. Instead, a change in phase was observed between the illuminated and the non-illuminated state of the SWCNT layer. The measured and simulated phase shifts are shown in Fig. 6.

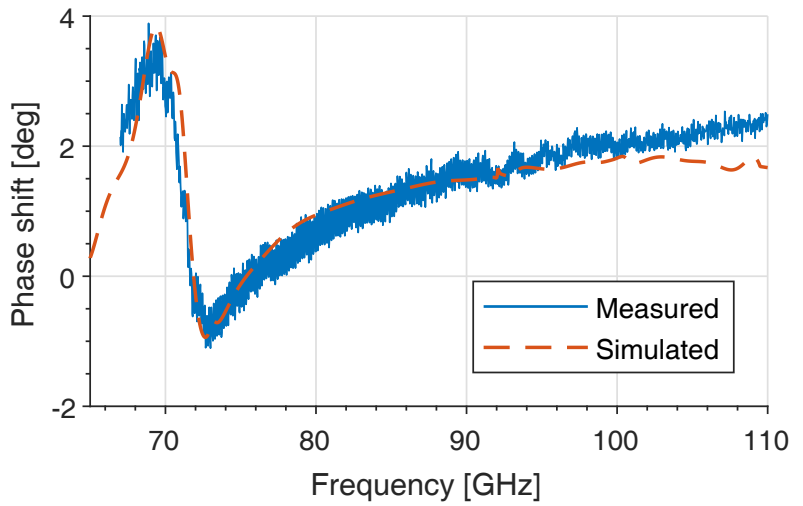

Fig. 6. Measured (plain curve) and simulated (dashed curve) phase shift in the DRW due to surface impedance variation by laser excitation of the SWCNTs.

A good agreement between them is observed with a clearly defined resonance peak around $72 \mathrm{GHz}$. This measurement allowed to extract an average nanotube length of $\approx 40 \mu \mathrm{m}$ in the sample, according to [15]. A slight difference in roll-off is present with a lower simulated phase shift than the measured one in the upper frequency range. This could be attributed to high frequency conductivity components of the SWCNTs in the $\mathrm{THz}$ range such as those described in [13] and [18]. They have not been modeled here, but can be added through additional oscillators in (1), to the detriment of a more complex model with a higher number of parameters to fit.

\section{CONCLUSION}

The approach presented in this paper allows efficient modeling of carbon nanotube layers in electromagnetic simulations of millimeter-wave devices such as phase shifters. To this end, a frequency-dependent impedance surface is calculated from the dielectric constant of the material. The proposed model keeps the simulation complexity low, as the impedance surface does not increase the total number of mesh cells, even for very thin material layers.

The correctness of the simulation model is verified by a good agreement with measured S-parameters' amplitude and phase shift of nanotube-covered dielectric rod waveguides in the W-band. The dielectric constant of the SWCNT layer was here analytically calculated by a Drude-Lorentz oscillator, accounting for the high losses in the lower frequency range and a resonant behavior of the optically-induced phase shift around $72 \mathrm{GHz}$. By further optimizing the SWCNTs properties, this approach could enable the design and simulation of wide-band, low-loss sub-THz phase shifters and other optoelectronic devices.

\section{ACKNOWLEDGMENT}

This work was financially supported in part by the European Union (EU) Horizon 2020 Innovative Training Network CELTA under grant agreement No 675683 of call: H2020-MSCA-ITN-2015, and by the European Research Council (ERC) under the European Union's Horizon 2020 
research and innovation programme (grant agreement No 616846).

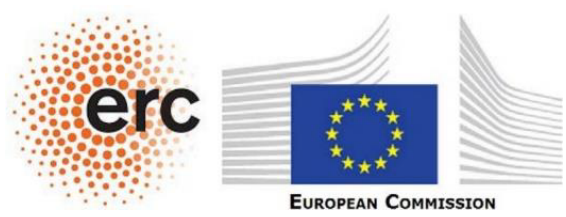

\section{REFERENCES}

[1] T. Kleine-Ostmann and T. Nagatsuma, "A review on terahertz communications research," J. Infrared Milli. Terahz Waves, vol. 32, no. 2, pp. 143-171, Jan. 2011.

[2] W. Menzel, "Millimeter-wave radar for civil applications," in 2010 European Radar Conf. (EuRAD). Paris, France: IEEE, 30 Sep.-1 Oct. 2010, pp. 89-92.

[3] J. El Haddad, B. Bousquet, L. Canioni, and P. Mounaix, "Review in terahertz spectral analysis," TrAC, Trends Anal. Chem., vol. 44, pp. 98-105, Mar. 2013.

[4] P. H. Siegel, "Terahertz technology in biology and medicine," IEEE Trans. Microw. Theory Techn., vol. 52, no. 10, pp. 2438-2447, Oct. 2004.

[5] D. Lioubtchenko, S. Tretyakov, and S. Dudorov, Millimeter-wave waveguides. New York: Springer Science \& Business Media, 2003.

[6] J. P. Pousi, S. N. Dudorov, D. V. Lioubtchenko, and A. V. Räisänen, "Frequency selective coupler for $\mathrm{W}$ band based on power transfer in dielectric rod waveguides," in Proc. 4th European Conf. Antennas and Propagation. Barcelona, Spain: IEEE, 12-16 Apr. 2010, pp. 1-4.

[7] J. P. Pousi, D. V. Lioubtchenko, S. N. Dudorov, and A. V. Raisanen, "High permittivity dielectric rod waveguide as an antenna array element for millimeter waves," IEEE Trans. Antennas Propag., vol. 58, no. 3, pp. 714-719, Mar. 2010.

[8] P. Pousi, D. Lioubtchenko, S. Dudorov, and A. V. Raisanen, "Dielectric rod waveguide travelling wave amplifier based on $\mathrm{AlGaAs} / \mathrm{GaAs}$ heterostructure," in 2008 38th European Microwave Conf. Amsterdam, Netherlands: IEEE, 27-31 Oct. 2008, pp. 1082-1085.

[9] P. J. Burke, "Carbon nanotube devices for $\mathrm{GHz}$ to $\mathrm{THz}$ applications," in Nanosensing: Materials and Devices, vol. 5593, International Society for Optics and Photonics. SPIE, Dec. 2004, pp. 52-62.
[10] J.-C. Charlier, X. Blase, and S. Roche, "Electronic and transport properties of nanotubes," Rev. Mod. Phys., vol. 79, no. 2, p. 677, May 2007.

[11] K. Gharbavi and H. Badehian, "Optical properties of armchair $(7,7)$ single walled carbon nanotubes," AIP Adv., vol. 5, no. 7, p. 077155, Jul. 2015.

[12] F. Borondics, K. Kamarás, M. Nikolou, D. Tanner, Z. Chen, and A. Rinzler, "Charge dynamics in transparent single-walled carbon nanotube films from optical transmission measurements," Phys. Rev. B, vol. 74, no. 4, p. 045431, Jul. 2006.

[13] G. Y. Slepyan, M. Shuba, S. Maksimenko, C. Thomsen, and A. Lakhtakia, "Terahertz conductivity peak in composite materials containing carbon nanotubes: Theory and interpretation of experiment," Phys. Rev. B, vol. 81, no. 20, p. 205423, May 2010.

[14] A. Maffucci, S. A. Maksimenko, G. Miano, and G. Y. Slepyan, "Electrical conductivity of carbon nanotubes: Modeling and characterization," in Carbon Nanotubes for Interconnects, A. Todri-Sanial, J. Dijon, and A. Maffucci, Eds. Springer, Jul. 2017, pp. 101-128.

[15] I. I. Nefedova, D. V. Lioubtchenko, I. S. Nefedov, and A. V. Räisänen, "Conductivity of carbon nanotube layers at low-Terahertz frequencies," IEEE Trans. THz Sci. Technol., vol. 6, no. 6, pp. 840-845, Nov. 2016.

[16] S. Maine, C. Koechlin, S. Rennesson, J. Jaeck, S. Salort, B. Chassagne, F. Pardo, J.-L. Pelouard, and R. Haïdar, "Complex optical index of single wall carbon nanotube films from the near-infrared to the terahertz spectral range," Appl. Opt., vol. 51, no. 15, pp. 3031-3035, May 2012.

[17] D. V. Lioubtchenko, I. V. Anoshkin, I. I. Nefedova, J. Oberhammer, and A. V. Räisänen, "W-band phase shifter based on optimized optically controlled carbon nanotube layer," in 2017 IEEE MTT-S Int. Microwave Symp. (IMS). Honololu, HI, USA: IEEE, 4-9 Jun. 2017, pp. 1188-1191.

[18] Q. Zhang, E. H. Hároz, Z. Jin, L. Ren, X. Wang, R. S. Arvidson, A. Lüttge, and J. Kono, "Plasmonic nature of the terahertz conductivity peak in single-wall carbon nanotubes," Nano Lett., vol. 13, no. 12, pp. 5991-5996, Nov. 2013. 